\title{
Digital Footprints in Drug Development: A Perspective from within the FDA
}

\author{
Interview with Dr. Sean Khozin, Acting Associate Director of the FDA \\ Oncology Center of Excellence
}

Dr. Sean Khozin is a thoracic oncologist and Acting Associate Director in the Food and Drug Administration's (FDA) Oncology Center of Excellence. He received his Doctor of Medicine from the University of Maryland School of Medicine and his Master of Public Health from George Washington University. Dr. Khozin is the Founding Director of FDA's Information Exchange and Data Transformation (INFORMED) and a member of the Editorial Board of Digital Biomarkers.

Digital Biomarkers: Today, I have the pleasure of speaking with Dr. Sean Khozin. Dr. Khozin, thank you very much for

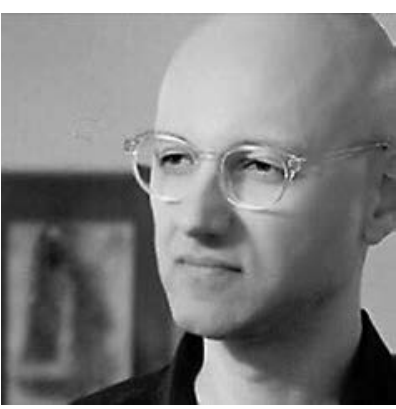

Dr. Sean Khozin joining us.

Dr. Khozin: Thanks, Ray, for having me.

Digital Biomarkers: Dr. Khozin, can you tell me a little about the FDA's Digital Health Innovation Action Plan?

Dr. Khozin: Appropriately validated digital health solutions, from smartphones to social media networks and web-based platforms, can empower patients and consumers to better manage their health. Emerging evidence suggests substantial benefits when these tools are implemented in clinical trials to capture the patients' experience outside the confines of the clinic. For example, in a recently published clinical trial involving 766 patients with metastatic solid tumors, a simple web-based questionnaire designed to track 12 common side effects related to anticancer therapy was associated with a 5-month improvement in overall survival. For these reasons, the FDA is exploring novel approaches to regulating digital health tools in order to facilitate efficient market entry of innovative technology products that can benefit patients and consumers. This approach, briefly outlined in the Digital Health Innovation Action Plan, is based on the recognition that traditional methods of regulating medical 
Fig. 1. Information Exchange and Data Transformation, also known as INFORMED.

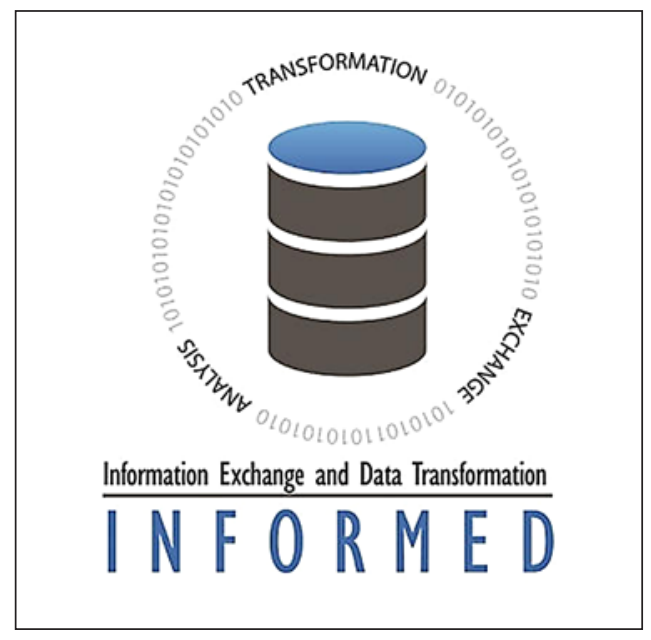

devices may not be suitable for the development and validation of modern digital health solutions. The FDA will announce further details about this program in the upcoming months.

Digital Biomarkers: You are the founding director of the Information Exchange and Data Transformation for the FDA. Can you tell us a little bit about that?

Dr. Khozin: Information Exchange and Data Transformation, also known as INFORMED, is a data science and technology incubator that we established at the FDA in collaboration with the Office of the Secretary at the Department of Health and Human Services (HHS) in late 2015 (Fig. 1). INFORMED was officially launched within the Office of Hematology and Oncology Products in May of 2016 under a special hiring authority to bring in entrepreneurs-in-residents from the private sector - Silicon Valley types, if you will.

INFORMED is designed and organized just like accelerators and incubators that help establish startups. Obviously, we're not commercializing products or launching companies. What we're doing is regulatory science with a forward-looking outlook based on principles of public entrepreneurship. We have a large portfolio of research collaborations and public/ private partnerships, all of which are focused on addressing specific public health and regulatory science questions related to oncology drug development and evidence generation.

When we established the Oncology Center of Excellence (OCE) earlier this year under the directorship of Dr. Richard Pazdur, INFORMED was folded into the new center as an official program within OCE.

Digital Biomarkers: I would love to know, what has been the most impactful work that has come out of INFORMED?

Dr. Khozin: We have two main areas of focus. One is focused on aggregating and standardizing clinical trial data that have been submitted to the FDA over the years. As you know, when sponsors of clinical trials submit a new drug application or biologic licensing application, they send us the clinical trial data for review. INFORMED has been aggregating this data and performing meta-analyses and predictive analytics. We've published some of this work and presented a number of abstracts at meetings such as the annual American Society of Clinical Oncology (ASCO) conference. We're testing, and in some cases developing, various technological solutions to streamline data curation and standardization. The results of some of our aggregated analyses are being used to inform internal decisions since not all of the work is in a suitable format for publication in peer-reviewed journals. However, findings that can have a substantial public health impact or are thought to be important to the drug development community are shared broadly through publications and our outreach efforts that include several public workshops each year. 
Digital Footprints in Drug Development: Interview with Dr. Sean Khozin

The second area of focus is going beyond clinical trial data for making regulatory decisions and we have several work streams in this category, a primary one being real-world evidence. Since the launch of INFORMED, we have engineered several partnerships and research collaborations to extract data from electronic health records. We have a research collaboration, for example, with CancerLinQ, a nonprofit subsidiary of ASCO, where we're examining the real-world experience of patients with advanced melanoma, with research objectives that include investigating the potential impact of how oncologists sequence new therapies approved by the FDA for advanced melanoma.

We also have a very productive collaboration with Flatiron Health and we presented the initial results of the effort at the 2017 ASCO annual meeting. With this collaboration, we are looking at the real-world experience of patients with metastatic, non-small cell lung cancer treated with immune checkpoint inhibitors. We are looking at patient characteristics and patterns of PD-L1 testing, which appear to be different from what we have observed in clinical trials. We are also looking at patient outcomes stratified by a variety of characteristics that can best be measured in the real world, such as history of autoimmune disease prior to receiving an immune checkpoint inhibitor.

Another area we're actively pursuing is leveraging opportunities to increase our digital footprint in drug development. For example, in collaboration with FDA's Office of Surveillance and Epidemiology (OSE), INFORMED developed a digital framework for transmission of safety information to the FDA, which is currently submitted as reports on paper and PDF files. This framework is now being piloted for implementation across the entire agency. Needless to say, transmission of safety information as datasets can lead to significant efficiencies in the detection of valid safety signals for industry, investigators, institutional review boards, and the FDA. As you could imagine, reading narratives on paper and PDF files is not even close to being an optimal solution for detecting patterns. Which brings us to this: why are we converting digital signals such as electrical activity captured by electrocardiograms to an analog format for human visual inspection?

Digital Biomarkers: Almost all the data collected in clinical trials comes from assessments conducted in clinics. What can real-world data add to our knowledge?

Dr. Khozin: Real-world data from the point of care can be a great opportunity to understand the experience of patients who are not involved in clinical trials. This is of particular importance in adult oncology where less than $5 \%$ of patients have an opportunity to participate in clinical studies. As a unique source of clinical evidence, real-world data can potentially support regulatory decisions to help clinicians tailor therapy to the individual needs of their patients, which is the hallmark of precision medicine.

We are currently working on developing a framework based on our research to support pragmatic clinical trials - studies conducted at the point of care using electronic health records as a conduit for data extraction. Pragmatic clinical trials are performed prospectively, and randomization can be applied if necessary.

Of course, not every question we want to answer would be suitable to test at the point of care in a pragmatic clinical trial, but the majority of empirical efforts outside of dose finding or mechanistic studies can be appropriate candidates for investigation through the prospective collection of real-world data.

Digital Biomarkers: What activities are you doing in the area of digital health?

Dr. Khozin: We're currently collaborating with the National Cancer Institute (NCI) on a pilot study to test the utility of biosensors to capture the experience of cancer patients. Among the variables we're collecting in this study are continuous metrics on performance status, which, despite being critical in determining patient eligibility for clinical trials and intensity of therapy in the real world, is still measured rather crudely by clinicians using the ECOG Performance Status or the Karnofsky Performance Scale. Our hope is to develop a digital 


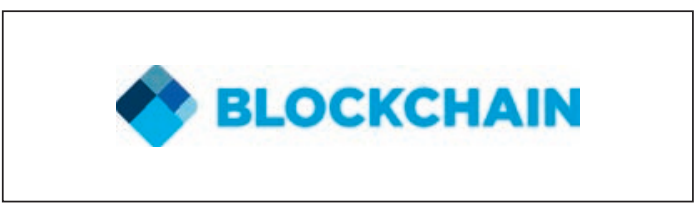

Fig. 2. Blockchain logo.

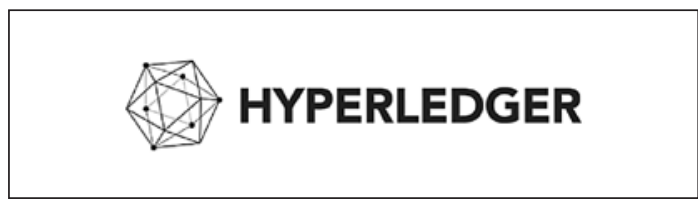

Fig. 3. Hyperledger logo.

biomarker for performance status based not only on patients' physical function, but also mood and cognition.

Digital Biomarkers: What biosensor are you using?

Dr. Khozin: We've assessed the lay of the land and have identified a digital solution for the study. We're not interested in simply acquiring a device for the study, but finding innovators who are interested in collaborating with us as scientists to develop novel digital biomarkers pre-competitively and for the benefit of cancer patients. Upon completion of appropriate agreements, we will announce our technology collaborators publicly.

Digital Biomarkers: Is it like a wearable accelerometer? Is it like a smartphone?

Dr. Khozin: It's a continuum of solutions consisting of a wearable device and a smartphone with specially designed apps to capture patient-reported outcomes to correlate with the sensor data and the clinical information captured at each visit. This is a joint effort with Dr. James Gulley at the NCI Center for Cancer Research, a national treasure for conducting cutting-edge clinical research which was the focus of a recent Discovery documentary series called First in Human. We're planning to do blood tests in the second stage of the pilot study to assess the correlation of digital biomarkers with blood-based counterparts.

Digital Biomarkers: One of the new technologies that you mentioned on your website is Blockchain. Have you had any applications of Blockchain technology in your work?

Dr. Khozin: Blockchain is part of our work stream for data sharing and data exchange (Fig. 2). We are working on testing the utility of Blockchain to enable a decentralized approach to data sharing at scale. As you know, Blockchain is used for the exchange of crypto currencies like Bitcoin. We are trying to see if we can take that framework and apply it to exchanging health data at scale among institutions, for example between the FDA and industry, and among patients and researchers. We would like to empower patients to exchange their data with anyone they want, including the research enterprise. Our Blockchain effort is a research collaboration with IBM Watson Health, a key contributor to the open source Hyperledger hosted by the Linux Foundation (Fig. 3). We've developed an alpha version of a Blockchain framework for health data exchange and are planning to publish the technical specifications early next year.

Other data-sharing efforts for INFORMED include co-sponsored symposia with Project Data Sphere (PDS), an open access platform for oncology clinical trial data. Studies conducted using PDS data have produced very intriguing results. For example, in a study recently published in Lancet Oncology, PDS led an effort where a prognostic model for prostate cancer was successfully developed through a crowdsourced Dream Challenge utilizing their data.

Digital Biomarkers: Dr. Khozin, you have an atypical background for an FDA regulator. You are an entrepreneur. You are an oncologist, and you are even a musician. How did you decide to come to the FDA, and what has it been like for someone like you?

Dr. Khozin: You're right, it is an unusual background that has enabled me to connect the dots (Fig. 4). We have a very unique window into the world of drug development and healthcare at the FDA, being at the convergence point of a lot of health data. We see what works and what doesn't work and we can often distinguish hype from reality. That can be very 
Fig. 4. Connecting the dots.

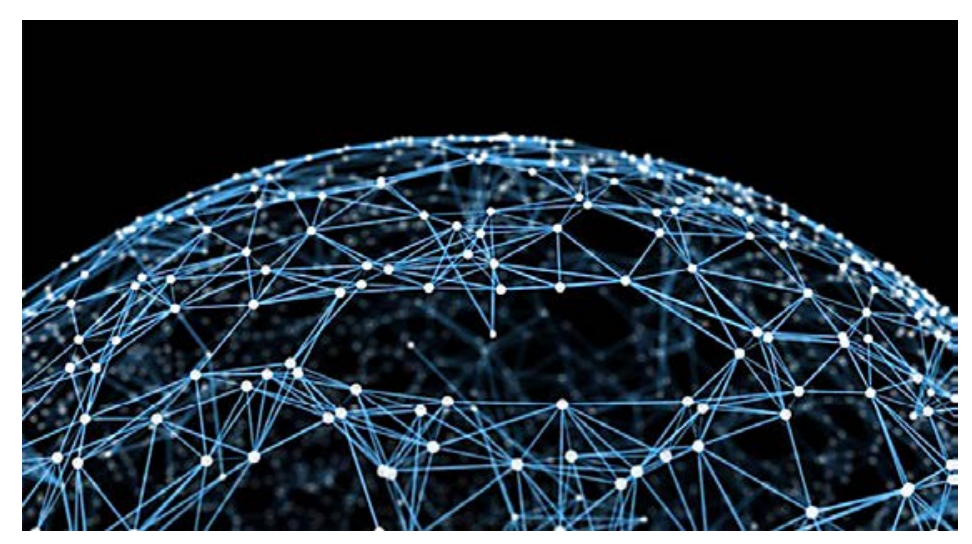

empowering, especially when coupled to an approach that is agile and entrepreneurial. The FDA is an organization full of smart and dedicated people and it's been a great privilege building INFORMED as a collaborative sound box with a singular vision focused on serving the interests of the public. Many of the projects in the INFORMED portfolio have never received any funding. They thrive on the wisdom of the crowd: amazing scientists at the FDA volunteering their time and the energy of our nation's endless pool of public and private sector innovators. I think we're at an inflection point in drug development and healthcare driven by a palpable atmosphere of enthusiasm and urgency that has unlocked opportunities for collaboration and outside-the-box thinking.

Digital Biomarkers: Dr. Khozin, thank you very much for all your efforts in advancing digital health within the FDA.

Dr. Khozin: Thank you, Ray. It's been a pleasure talking to you. 